\title{
Analysis of the Main Aspects of Testing the Opaque Version of CAPM
}

\author{
Constantin Anghelache ${ }^{1}$, Dana Luiza Grigorescu², Marius Popovici ${ }^{3}$ \\ ${ }^{1}$ The Bucharest University of Economic Studies/„Artifex” University of Bucharest, Romania, \\ ${ }^{1}$ E-mail: actincon@yahoo.com \\ ${ }^{2,3}$ Bucharest University of Economic Studies, Romania, \\ 2E-mail: danaliza2004@yahoo.com, 33E-mail: popovicidumitrumarius@gmail.com
}

\begin{abstract}
The opaque version of the capital asset pricing model (CAPM) is a little more complicated in terms of the restrictions it imposes on the data series. In this context there is a cross-equation constraint for the null hypothesis. As a rule, a resected model should be considered and estimated as nonlinear. The model implies the condition that the estimators obtained and the estimation are usually nonlinear. The estimation procedure is nonlinear, but to simplify it we resort to calculating the particular structures that are conditioned and are obtained from a linear regression. Simple linear regression in this case or multiple is the one that ensures obtaining parameters that are efficient in estimating the respective results. The authors were concerned with the analysis of the main aspects regarding the testing of the opaque version of the CAPM, considering that the cross-regression tests must be taken into account, considering in this regard the approach with two regression functions introduced by Fama and MacBeth (1973) and the case SharpeLintner.

Key words Methods, Models, Assets, Capital Market, Estimators, Regressions, Statistical Tests

Received: 12 Mar 2020 (C) The Authors 2020

Revised: 20 Mar 2020 Published by Human Resource Management Academic Research Society (www.hrmars.com)

Accepted: 26 Mar 2020 This article is published under the Creative Commons Attribution (CC BY 4.0) license. Anyone may Published Online: 07 Apr 2020 reproduce, distribute, translate and create derivative works of this article (for both commercial and non-commercial purposes), subject to full attribution to the original publication and authors. The full terms of this license may be seen at: http://creativecommons.org/licences/by/4.0/legalcode
\end{abstract}

\section{Introduction}

In the article, the main aspects regarding the testing of the opaque version of the CAPM are presented several approaches and, if one use the OLS statistics, two approaches are possible. A first possibility of approach is presented by the null hypothesis, where the vector with typical elements exists. For the implementation of this test, the variation of the sample is calculated and further it results that in the large samples, under the null hypothesis, a most consistent probability results. The second approach is the one that is performed after several iterations, in the sense that, after finding an analytical expression for the distribution of these test statistics, under normal conditions, simulation models could be used to obtain an exact distribution below the same hypothesis of normality, and another alternative method of calculation is that used by Walt, in which the matrix of estimated covariance is unrestricted.

The robustness to normality and heterostedasticity of the time series is an important element that is addressed, in the light of how the CAPM model can offer some solutions, sometimes weaker than under normal conditions. Important is also the fact that some cross-regression tests are carried out, in which two regression functions are introduced which is widely used due to the ease of solving and implementation. In the case of such an approach, a linear connection with an equal slope of the excessive average yield on the 
market must be ensured. We can appreciate that this is a simple linear regression adapted to the conditions of the market study mentioned above.

Empirically it follows that there is a positive and linear relationship between the risks of high yield, with a lower significance in the case of excessive market yield. Data were used to highlight this evolution trend.

\section{Literature review}

Ameur \& Prigent (2010) analyzed the typology of risk behavior in portfolio management. Ang et al. (2006) they addressed issues related to estimating yields and volatility. Anghel (2013) highlighted the main methods and models used in portfolio analysis. Anghelache et al. (2019) have analyzed a number of elements of dynamic models. Anghelache et al. (2016a) they studied fundamental issues regarding the management of the portfolio of shares. Anghelache (2008) highlighted the statistical tool used in the economic analyzes. Anghelache et al. (2016b), as well as Campbell and Viceira (2002) researched the main elements of portfolio selection. Chen \& Ang (2007) and Grauer \& Janmaat (2009) analyzed the results of applying the CAPM model. Gollier (2011) \& Wolff (2013) they paid more attention to the price of assets. lacob et al. (2020), as well as Wachter (2002) presented issues regarding the selection and decisions taken regarding financial instrument portfolios. Tetlock (2011) referred to the role of information in the decisionmaking process. Wooldrige (2006) presented the importance of using econometric models in economic studies. Zhou (2010) analyzed issues regarding the forecast of the profitability of the shares.

\section{Methodology of research. Results and discussions}

The opaque version of the CAPM is more complicated in terms of the restrictions it imposes on data. There is a cross equation constraint under the conditions of the null hypothesis. The restricted model to be estimated is nonlinear. The model to be built is estimated under the variant, that before using the total yields we use excess yields.

The model involving the above condition is of the form:

$\alpha=\left(i_{N}-\beta\right) \gamma$

for an unknown scalar parameter $\psi$, where $i_{N}$ is a vector $N$ of the primaries. We can rewrite this equation by highlighting the $\mathrm{N}-1$ constraints of the nonlinear cross equation involved, in the form:

$$
\frac{\alpha_{1}}{1-\beta_{1}}=\cdots=\frac{\alpha_{N}}{1-\beta_{1}}=\gamma
$$

We mention that it takes at least two assets to test the opaque version, because otherwise we can always find a scalar $(\gamma)$, such that $\gamma=\frac{\alpha}{1-\beta}$. When $N \geq 2$, this ratio must be the same for all assets, which is a testable restriction. Estimating the maximum likelihood of the model to be built is equivalent to OLS of, equation after equation, with the gross returns instead of the excess returns. For $\theta=\left(\gamma, \beta_{1}, \ldots, \beta_{N}\right)^{T}$ the probability function $R_{1}, \ldots, R_{T}$, respectively $R_{m 1}, \ldots, R_{m T}$ are form:

$$
\ell\left(\theta, \Omega_{s}\right)=c-\frac{T}{2} \log \operatorname{det}\left(\Omega_{s}\right)-\frac{1}{2} \sum_{t=1}^{T}\left[R_{t}-\gamma i_{N}-\beta\left(R_{m t}-\gamma\right)\right]^{T} \Omega_{s}^{-1}\left[R_{t}-\gamma i_{N}-\beta\left(R_{m t}-\gamma\right)\right]
$$

Where $c$ is a constant that does not depend on unknown parameters. MLE from ${ }^{\theta,} \Omega_{\varepsilon}$ which maximizes $\ell\left(\theta, \Omega_{s}\right)$ is noted $\widehat{\theta}^{*}, \widehat{\Omega}_{s}^{*}$. The estimation procedure is nonlinear but we can simplify the calculation by observing the particular structure that conditions on $\gamma$ and we have a linear regression, defined for each one ${ }^{Y \in R}$, using the relationship:

$$
\begin{aligned}
& \hat{\beta}_{i}^{*}(\gamma)=\frac{\sum_{t=1}^{T}\left(R_{m t}-\gamma\right)\left(R_{\mathrm{it}}-\gamma\right)}{\sum_{t=1}^{T}\left(R_{m t}-\gamma\right)^{2}} \\
& \hat{\Omega}_{s}^{*}(\gamma)=\frac{1}{T} \hat{\varepsilon}^{*}(\gamma) \hat{\varepsilon}^{T}(\gamma)=\left[\frac{1}{T} \sum_{t=1}^{T} \hat{\varepsilon}_{i t}^{*}(\gamma) \hat{\varepsilon}_{j t}^{*}(\gamma)\right]_{i j}
\end{aligned}
$$


Then we determine the profile probability or the concentrated probability, by the equation:

$\ell_{p}(\gamma)=c-\frac{T}{2} \log \operatorname{det}\left[\hat{\Omega}_{s}^{*}(\gamma)\right]-\frac{1}{2} \sum_{t=1}^{T}\left[R_{t}-\gamma i_{N}-\hat{\beta}^{*}\left(R_{m t}-\gamma\right)\right]^{T} \Omega_{s}^{-1}\left[R_{t}-\gamma i_{N}-\hat{\beta}^{*}\left(R_{m t}-\gamma\right)\right]_{(5)}$

above the scalar parameter ${ }^{\gamma}$. The value ${ }^{\gamma}$ which maximizes $\ell_{p}(\gamma)$ it is noted $\hat{\gamma}_{p}$. Then we define: $\hat{\beta}_{p}^{*}=\hat{\beta}^{*}\left(\hat{\gamma}_{p}\right) ; \widehat{\Omega}_{s p}^{*}=\widehat{\Omega}_{s}^{*}\left(\hat{\gamma}_{p}\right)$

In fact, it can be shown that $\hat{\beta}_{p}^{*}=\hat{\beta}^{*}{ }_{\text {si }} \widehat{\Omega}_{s p}^{*}=\widehat{\Omega}_{s}^{*}$, so this calculation device works.

At this moment we can calculate the test of the probability ratio after the relation:

$L R_{B}=T\left[\log \operatorname{det}\left(\widehat{\Omega}_{s}^{*}\right)-\log \operatorname{det}\left(\widehat{\Omega}_{s}\right)\right]$

where $\widehat{\Omega}_{s}^{*}$ it is MLE of ${ }^{\Omega} s_{s}$ in the constrained model. It satisfies the relationship:

$L R_{B}=>x_{N-1}^{2}$

with ${ }^{T \rightarrow \infty}$ under the null hypothesis.

The exact distribution is unknown $L R_{B}$. Currently, simulation methods are practical ways to approach this issue and we will continue to establish a simple algorithm to obtain the distribution of test statistics. If $\varepsilon^{*} \sim N\left(0, \widehat{\Omega}_{\varepsilon}^{*}\right)$ a new sample is set for $t=1, \ldots, T$, results:

$R_{t}^{*}=\left(i_{N}-\hat{\beta}^{*}\right) \hat{y}_{p}+\hat{\beta}^{*} R_{m t}+\varepsilon_{t}^{*}$

We also determine for this sample the test statistic, respectively:

$L R_{B}^{*}=T\left[\log \operatorname{det}\left(\widehat{\Omega}_{s}^{*}\right)-\log \operatorname{det}\left(\hat{\Omega}_{s}\right)\right]$

which can be repeated $B$ times.

Next we use the empirical distribution of $B$ in the test statistics $L R_{B}^{*}$ to define the critical value $c_{\alpha}^{*}$ of the test we reject if $L R_{B}$ initially exceeds $c^{*}$. Suppose it can be substituted $\varepsilon^{*} \sim N\left(0, \widehat{\Omega}_{s}^{*}\right)$ starting from the empirical distribution of errors (Efron 1979).

If we consider Wald statistics, we identify two approaches. In the first we present the null hypothesis with $g=0$, where ${ }^{g}$ is the vector ${ }^{N-1 \times 1}$ with typical element:

$g_{i}=\frac{\alpha_{i}}{1-\beta_{i}}-\frac{\alpha_{N}}{1-\beta_{N}}, \quad i=1, \ldots, N-1$

Whether

$\hat{g}=\left(\hat{g}_{1}, \ldots, \hat{g}_{N-1}\right)^{T}$, unde

$\widehat{g}_{i}=\frac{\widehat{\alpha}_{i}}{1-\widehat{\beta}_{i}}-\frac{\widehat{\alpha}_{N}}{1-\widehat{\beta}_{N}}, \quad i=1, \ldots, N-1$

To implement this test we need to calculate the variation of the vector sample ${ }^{g}$. If $\phi=\left(\alpha^{T}, \beta^{T}\right)^{T}$, $\widehat{\phi}=\left(\hat{\alpha}^{T}, \hat{\beta}^{T}\right)^{T}, g=G(\phi)$ and $\hat{g}=G(\hat{\phi})$, where $G: \mathbb{R}^{2 N} \rightarrow \mathbb{R}^{N-1}$ is a known function, we will use the relationship: 
$\Gamma(\phi)=\frac{\partial G}{\partial \phi^{T}}=\left(\begin{array}{ccc}\frac{\partial G_{1}}{\partial \alpha_{1}} & \cdots & \frac{\partial G_{1}}{\partial \beta_{N}} \\ \frac{\partial G_{N-1}}{\partial \alpha_{1}} & \cdots & \frac{\partial G_{N-1}}{\partial \beta_{N}}\end{array}\right)=\left[\begin{array}{cccc}\frac{1}{1-\beta_{1}} & 0 \ldots \frac{-1}{1-\beta_{N}} & -\frac{\alpha_{1}}{\left(1-\beta_{1}\right)^{2}} & 0 \ldots \frac{\alpha_{N}}{\left(1-\beta_{N}\right)^{2}} \\ \frac{1}{1-\beta_{N-1}} & 0 \ldots \frac{-1}{1-\beta_{N}} & -\frac{\alpha_{N-1}}{\left(1-\beta_{N-1}\right)^{2}} & 0 \ldots \frac{\alpha_{N}}{\left(1-\beta_{N}\right)^{2}}\end{array}\right]$

$V_{g}=\Gamma(\phi)\left[\begin{array}{cc}\left(1+\frac{\mu_{m}^{2}}{\sigma_{m}^{2}}\right) \Omega_{s} & \frac{-\mu_{m}}{\sigma_{m}^{2}} \Omega_{s} \\ \frac{-\mu_{m}}{\sigma_{m}^{2}} \Omega_{s} & \frac{1}{\sigma_{m}^{2}} \Omega_{s}\end{array}\right] \Gamma(\phi)^{T}$

Where $\Gamma(\phi)$ it is $N-1 \times 2 N$ the matrix of partial derivatives $\mu_{m}=E\left(R_{m}\right)$ and $\sigma_{m}^{2}=\operatorname{var}\left(R_{m}\right)$. We will use the delta method and it will turn out that in large samples with ${ }^{T \rightarrow \infty}$, we will have:

$\sqrt{T}(\hat{g}-g)=>N\left(0, V_{g}\right)$

Whether

$W_{B}=\hat{g} \hat{V}_{g}^{-1} \hat{g}$

$\hat{V}_{g}=\widehat{\Gamma}\left[\begin{array}{cc}\left(1+\frac{\bar{z}_{m}^{z}}{\hat{\sigma}_{m}^{2}}\right) \hat{\Omega}_{s} & \frac{-\bar{z}_{m}}{\widehat{\sigma}_{m}^{2}} \widehat{\Omega}_{s} \\ \frac{-\bar{z}_{m}}{\hat{\sigma}_{m}^{2}} \widehat{\Omega}_{s} & \frac{1}{\hat{\sigma}_{m}^{2}} \widehat{\Omega}_{s}\end{array}\right] \widehat{\Gamma}^{T}$

where $\hat{\Gamma}=\Gamma(\widehat{\phi})$. Thus, it turns out when ${ }^{T \rightarrow \infty}$ leads to:

$W_{B}=>x_{N-1}^{2}$

under the null hypothesis.

This is solved by several iterations. It is not possible to find analytical expressions for the distribution of this test statistic under normal conditions, although simulation methods could be used to obtain an exact distribution under the normality hypothesis. An alternative way of calculating Wald statistics is as follows, using the relation:

If $\delta=\left(\gamma, \beta_{1}, \ldots, \beta_{N}\right)^{T} \in \mathbb{R}^{N+1}$ results in the restricted parameters and $h(\delta)=\left[\gamma\left(1-\beta_{1}\right), \beta_{1}, \ldots, \gamma\left(1-\beta_{N}\right), \beta_{N}\right]^{T} \in \mathbb{R}^{2 N}$. Then:

$W=T \min _{\delta \in \mathbb{R}^{N+1}}[\hat{\theta}-h(\delta)]^{T} \widehat{\pi}^{-1}[\hat{\theta}-h(\delta)]$

If $\widehat{\pi}=\widehat{\Omega}_{s} \Pi\left(X^{T} X\right)^{-1}$ it is ${ }^{2 N \times 2 N}$ the estimated covariance matrix of the unrestricted estimates ${ }^{\hat{\theta}}$. The symbol $\Pi$ represents the Kronecker product of two matrices.

In Chamberlain's acceptance, under the null hypothesis $W=>x_{N-1}^{2}$ and $T \rightarrow \infty$, while under alternative hypothesis $W$ grows without limits.

We can explicitly solve for ${ }^{\gamma}$, respectively:

$\gamma=\frac{(i-\beta)^{T} \Omega_{\varepsilon}^{-1} \alpha}{(i-\beta)^{T} \Omega_{\varepsilon}^{-1}(i-\beta)}$

Which shows that we also have, ${ }^{\beta \neq i}$ for this restriction to make sense. ${ }^{\gamma}$ estimated is:

$\hat{\gamma}=\frac{(i-\widehat{\beta})^{T} \widehat{\hat{\Omega}}_{\varepsilon}^{-1} \widehat{\alpha}}{(i-\widehat{\beta})^{T} \widehat{\widehat{\Omega}_{\varepsilon}^{-1}}(i-\widehat{\beta})}$

and measures the performance of the beta portfolio zero.

The capital asset pricing model (CAPM) may offer solutions that are weaker than normal. Maximum likelihood estimation involves normal multivariate returns (otherwise, it is called quasi-maximum likelihood estimator QMLE probability). In fact, the QMLEs in $\alpha, \beta$ they are robust to heterostedestaticity, serial correlation and non-normality, because precision requires only the correct specification of the mean. There 
is no need to invoke the GMM concept to address this application problem. However, the exact distribution theory is no longer valid when normality is not maintained. In addition, large sample theory for test statistics should be considered. Specifically, it is necessary to adjust the standard errors for time series heterostedestaticity, a problem solved by the regression models and within the quasi-probability framework long ago.

If we assume that the error term is given by a martingale difference sequence, we obtain:

$E\left(\varepsilon_{t} \mid F_{t-1}, Z_{m 1}, \ldots, Z_{m T}\right)=0$

$E\left(\varepsilon_{t} \varepsilon_{t}^{T} \mid F_{t-1}, Z_{m 1}, \ldots, Z_{m T}\right)=\Omega_{s t}$

where $\Omega_{s t}$ is a potentially random covariance matrix (depending on $F_{t-1}, Z_{m t}$ ) and $F_{t-1}$ represents all prior information on returns, including $Z_{m t}$. It is a general approach, but as we will see, it is quite natural to allow dynamic heterostedestaticity for data on stock returns. We consider models for ${ }^{s t}$ and we will allow them to vary freely over time.

We consider the average covariance matrices, from the relations:

$\bar{\Omega}_{s T}=\frac{1}{T} \sum_{t=1}^{T} \Omega_{s t} ; \quad \Omega_{T}=\frac{1}{T} \sum_{t=1}^{T}\left(Z_{m t}-\bar{Z}_{m}\right)^{2} \Omega_{s t}$

Assuming that they have positive limits defined and finite ${ }^{T \rightarrow \infty}$. We can build robust Wald tests and LM tests based on large sample approximations that approach normality assumptions. Specifically, considering relationships:

$\widehat{\Omega}_{H}=\frac{1}{T} \sum_{t=1}^{T}\left[1+\frac{\bar{z}_{m}^{2}}{\hat{\sigma}_{m}^{4}}\left(Z_{m t}-\bar{Z}_{m}\right)^{2}\right] \hat{\varepsilon}_{t} \hat{\varepsilon}_{t}^{T}$

$\tilde{\Omega}_{H}=\frac{1}{T} \sum_{t=1}^{T}\left(1-\frac{\bar{z}_{m}}{\hat{\mu}_{m z}} Z_{m t}\right)^{2} \tilde{\varepsilon}_{t} \tilde{\varepsilon}_{t}^{T}$

and then the $\mathrm{W}$-robust Wald statistics and the $\mathrm{H}$-robust LM statistics, given by the relationships:

$W_{H}=T \hat{\alpha} \widehat{\Omega}_{H}^{-1} \hat{\alpha}_{;} \quad L M_{H}=T \overline{\tilde{\varepsilon}} \tilde{\Omega}_{H}^{-1} \overline{\tilde{\varepsilon}}$

In accordance with the null hypothesis and some additional conditions in the form ${ }^{\Omega}$, but which does not require normality, with ${ }^{T \rightarrow \infty}$

$W_{H}, L M_{H}=>x_{N}^{2}$

According to the alternative hypothesis, it turns aut $W_{H}, L M_{H} \rightarrow \infty$

Fama and MacBeth (1973) introduced the two-step regression approach, which is very popular due to its easy solution and implementation. This approach underlines the cross-cutting implications of CAPM. In the case of Sharpe-Lintner, the form with excessive yield $E_{i}=E\left(R_{i}-R_{f}\right)$ should be linearly related to $\beta_{i}$ with a slope equal to the excessive average yield on the market $E_{m}=E\left(R_{m}-R_{f}\right)$. In the opaque case the average efficiency of the company $\mu_{i}=E\left(R_{i}\right)$ should be linearly related to ${ }^{\beta_{i}}$ with the transverse value $E\left(R_{0}\right)$ and a slope equal to the excessive average yield on the market compared to the zero beta portfolio, $E\left(R_{m}-R_{0}\right)$. If we focus on the opaque case, the null model for yields is given by the relation:

$R_{i t}=\gamma_{0 t}+\beta_{i} \gamma_{1 t}+e_{i t}$

where $\gamma_{0 t}=R_{0 t}$ is the yield of the zero beta portfolio, provided $\gamma_{1 t}=R_{m t}-R_{0 t}$. 
If $\Gamma_{t}=\left(\gamma_{0 t}, \gamma_{1 t}\right)^{T}, t=1, \ldots, T$, suppose that $e_{i t}$ satisfy $E\left(e_{i t} \mid \Gamma_{1}, \ldots, \Gamma_{t}\right)=0$ and $E\left(e_{i t} e_{i j} \mid \Gamma_{1}, \ldots, \Gamma_{t}\right)=\sigma_{i j}, t=s$ provided it is zero. Otherwise, the relationship becomes:

$\bar{R}_{i}=\bar{\gamma}_{0}+\beta_{i} \bar{\gamma}_{1}+\bar{e}_{i}$

This leads to the conclusion that it indicates the serial media, as usual, $\left(\bar{R}_{i}=\sum_{t=1}^{T} \frac{R_{\mathrm{it}}}{T}\right.$ ). This becomes a cross-sectional regression with covariates consisting of the unit vector. The errors in this regression are small (due to the average time series), but correlated. We will consider $X=\left(X_{1}, \ldots, X_{N}\right)^{T}$, where $X_{i}=\left(1, \beta_{i}\right)^{T}, \bar{R}=\left(\bar{R}_{1}, \ldots, \bar{R}_{N}\right)^{T}$ and $\bar{\Gamma}=\left(\bar{\gamma}_{0}, \bar{\gamma}_{1}\right)^{T}$.

OLS estimator of $\bar{\Gamma}$ is given by the relationship:

$\bar{\Gamma}=\left(X^{T} X\right)^{-1} X^{T} \bar{R}$

Parameter $\bar{\Gamma}$ is the average of the time series $\Gamma_{t}=\left(\gamma_{0 t}, \gamma_{1 t}\right)^{T}$, and the parameter of interest is $\Gamma=\mathrm{E}\left(\Gamma_{t}\right)$. Therefore, it turns out:

$\widetilde{\Gamma}-\Gamma=\widetilde{\Gamma}-\bar{\Gamma}+\bar{\Gamma}-\Gamma=\left(X^{T} X\right)^{-1} X^{T} \bar{e}+\bar{\Gamma}-\Gamma$

which has two sources of variation. Suppose ${ }^{\Gamma_{t}}$ is a stationary martingale difference sequence, with:

$\pi=E\left[\left(\Gamma_{t}-\Gamma\right)\left(\Gamma_{t}-\Gamma\right)^{T}\right]$

defined positive, resulting:

$\operatorname{var}(\tilde{\Gamma}-\Gamma)=\frac{1}{T}\left[\left(X^{T} X\right)^{-1} X^{T} \Omega_{s}\left(X^{T} X\right)^{-1}+\pi\right]$

and

$\sqrt{T}(\widetilde{\Gamma}-\Gamma)=>N(0, \psi)$

where $^{\psi}=\left(X^{T} X\right)^{-1} \Omega_{s} X\left(X^{T} X\right)^{-1}+\Sigma$. The question that arises is how to obtain an estimate ${ }^{\psi}$. The FM approach is next. Whether:

$\tilde{\Gamma}_{t}=\left(X^{T} X\right)^{-1} X^{T} R_{t}$

where $\quad R_{t}=\left(R_{1 t}, \ldots, R_{N t}\right)^{T}=X \Gamma_{t}+\mathrm{e}_{t}, \quad \mathrm{e}_{t}=\left(e_{1 t}, \ldots, e_{N t}\right)^{T}$. It turns out that $\tilde{\Gamma}_{t}-\Gamma_{t}=\left(X^{T} X\right)^{-1} \mathrm{e}_{t}+\Gamma_{t}-\Gamma$. It `s being suggested:

$\tilde{\psi}=\frac{1}{T} \sum_{t=1}^{T}\left(\tilde{\Gamma}_{t}-\tilde{\Gamma}\right)\left(\tilde{\Gamma}_{t}-\tilde{\Gamma}\right)^{T}=\frac{1}{T} \sum_{t=1}^{T}\left(X^{T} X\right)^{-1} X^{T}\left[\left(R_{t}-\bar{R}\right)\left(R_{t}-\bar{R}\right)^{T}\right] X\left(X^{T} X\right)^{-1}=$

$\left(X^{T} X\right)^{-1} X^{T}\left[\frac{1}{T} \sum_{t=1}^{T}\left(R_{t}-\bar{R}\right)\left(R_{t}-\bar{R}\right)^{T}\right] X\left(X^{T} X\right)^{-1}$

and we have $R_{t}-\bar{R}=X\left(\Gamma_{t}-\bar{\Gamma}\right)+\mathrm{e}_{t}-\bar{e}$, so, we can end the relationship:

$\frac{1}{T} \sum_{t=1}^{T}\left(R_{t}-\bar{R}\right)\left(R_{t}-\bar{R}\right)^{T}=\frac{1}{T} \sum_{t=1}^{T}\left[X\left(\Gamma_{t}-\bar{\Gamma}\right)+\left(e_{t}-\bar{e}\right)\right]\left[X\left(\Gamma_{t}-\bar{\Gamma}\right)+\left(e_{t}-\bar{e}\right)\right]^{T}=$

$x\left[\frac{1}{T} \sum_{t=1}^{T}\left(\Gamma_{t}-\bar{\Gamma}\right)\left(\Gamma_{t}-\bar{\Gamma}\right)^{T}\right] X^{T}+\frac{1}{T} \sum_{t=1}^{T}\left(e_{t}-\bar{e}\right)\left(e_{t}-\bar{e}\right)^{T}+X \frac{1}{T} \sum_{t=1}^{T}\left(\Gamma_{t}-\bar{\Gamma}\right)\left(e_{t}-\bar{e}\right)^{T}+$

$\frac{1}{T} \sum_{t=1}^{T}\left(e_{t}-\bar{e}\right)\left(\Gamma_{t}-\bar{\Gamma}\right)^{T} X^{T}$ 
Provided that $e_{t}$ is exogenous in relation to the process $\Gamma_{t}$, the cross terms of the product converge in probability to zero and $T \rightarrow \infty$, get:

$\tilde{\psi} \stackrel{p}{\rightarrow} \psi$

We will consider a more general case that allows testing of CAPM restrictions. Let's suppose that ${ }^{S_{i}}$, is another measure of risk. Then for each period $\sigma_{s i}$ we can introduce, the relation (28) in the general regression model, of the form:

$Z_{i t}=\gamma_{0 t}+\gamma_{1 t} \beta_{i}+\gamma_{2 t} \beta_{i}^{2}+\gamma_{3 t} S_{i}+e_{i t}$

where the parameters $\gamma_{j t}$ are the best coefficients of linear predictors of $Z_{i t}$.

If we assume that $\Gamma_{t}=\left(\gamma_{0 t}, \gamma_{1 t}, \gamma_{2 t}, \gamma_{3 t}\right)^{T}$ is a stationary process with finite variation and $\Gamma_{t}-\Gamma_{\text {is a }}$ sequence of marginal difference, where $\Gamma=E\left(\Gamma_{t}\right)$, we consider that ${ }^{e_{i t}}$ satisfy $E\left(\mathrm{e}_{i t} \mid \Gamma_{1}, \ldots, \Gamma_{T}\right)=0$ and $E\left(\mathrm{e}_{i t} \mathrm{e}_{j s} \mid \Gamma_{1}, \ldots, \Gamma_{T}\right)=\sigma_{i j}$ if $t=s$ or zero otherwise.

CAPM Shrpe-Lintner with $Z_{i t}=R_{i t}-R_{f t}$ consider that $E\left(\gamma_{0 t}\right)=E\left(\gamma_{1 t}\right)=E\left(\gamma_{2 t}\right)=E\left(\gamma_{3 t}\right)$ and $\gamma_{1 t}=Z_{m t}=R_{m t}-R_{f t}$. Opaque CAPM-up implies that $E\left(\gamma_{2 t}\right)=E\left(\gamma_{3 t}\right)=0$ and $E\left(\gamma_{0 t}\right)$ is the average of the zero-beta asset, while $E\left(\gamma_{1 t}\right)$ is equal to the average excess yield on the market compared to the zero beta portfolio.

Suppose $\beta_{i}$ and $s_{i}$ are known. With $\mathrm{X}=\left(X_{1}, \ldots, X_{N}\right)^{T}$, where $X_{i}=\left(1, \beta_{i}, \beta_{i}^{2} \cdot \sqrt{\sigma_{i i}}\right)^{T}$ and either $\mathrm{Z}_{t}=\left(Z_{1 t}, \ldots, Z_{N t}\right)^{T}$ and $\bar{Z}=T^{-1} \sum_{t=1}^{T} \mathrm{Z}_{t}$. Under the null hypothesis, it turns out:

$Z_{t}=X \Gamma_{t}+e_{t}, \quad \bar{Z}=X \bar{\Gamma}+\overline{\mathrm{e}}$

where $\bar{\Gamma}=T^{-1} \sum_{t=1}^{T} \Gamma_{t}$.

We will consider:

$\tilde{\Gamma}_{t}=\left(X^{T} W X\right)^{-1} X^{T} W Z_{t} ; \quad \tilde{\Gamma}=\left(X^{T} W X\right)^{-1} X^{T} W \bar{Z}=\frac{1}{T} \sum_{t=1}^{T} \tilde{\Gamma}_{t}$

Where $W$ it is ${ }^{N x N}$ positive symmetrical matrix defined positive. We will share with $W=I_{N}$ based on cross-section correlation and heterostedestaticity in errors. To evaluate $\widetilde{\Gamma}^{\tilde{}}$ (the estimator), first we will condition $\Gamma_{1}, \ldots, \Gamma_{T}$ and then we will consider the average over the distribution of these random variables. We can write the relation (39), equivalently, in the form:

$Z_{t}=X \Gamma+u_{t}, u_{t}=e_{t}-X\left(\Gamma_{t}-\Gamma\right)$

as a random variable not correlated with ${ }^{e_{t}}$, considering:

$\pi=E\left[\left(\Gamma_{t}-\Gamma\right)\left(\Gamma_{t}-\Gamma\right)^{T}\right]$

which becomes a covariance matrix.

Under the null hypothesis defined $E\left(\tilde{\Gamma}_{t} \mid \Gamma_{1}, \ldots, \Gamma_{T}\right)=\Gamma_{t}$ and $E\left(\tilde{\Gamma}_{t} \mid \Gamma_{1}, \ldots, \Gamma_{T}\right)=\frac{1}{T} \sum_{t=1}^{T} \Gamma_{t}$, then we will have the relationship:

$\psi=\operatorname{var}\left(\widetilde{\Gamma}_{t}\right)=\left(X^{T} W X\right)^{-1} X^{T} W \Omega_{s} X\left(X^{T} W X\right)^{-1}+\pi$

and $\operatorname{var}(\widetilde{\Gamma})=\operatorname{var}\left(\tilde{\Gamma}_{t}\right) / T$. In addition we consider the relationship:

$\sqrt{T}(\tilde{\Gamma}-\Gamma)=>N(0, \psi), \mathrm{cu}^{T \rightarrow \infty}$

We also have

$\widetilde{\psi}=\frac{1}{T} \sum_{t=1}^{T}\left(\tilde{\Gamma}_{t}-\tilde{\Gamma}\right)\left(\widetilde{\Gamma}_{t}-\tilde{\Gamma}\right)^{T} \stackrel{p}{\rightarrow} \psi$ 
and $T \rightarrow \infty$. Considering the hypothesis $Q \Gamma=q \in \mathbb{R}^{2}$, where $Q, q$ are known arrays. Wald statistics satisfies the relationship:

$T(Q \tilde{\Gamma}-q)^{T}\left(Q \widetilde{\psi} Q^{T}\right)^{-1}(Q \tilde{\Gamma}-q)=>x_{p}^{2}$

In practice, we do not consider ${ }^{\beta_{i}}$ and $S_{i}$, which we replace by considered estimates. Suppose $S_{i}=\sqrt{\sigma_{i i}}$ then (for the Sharpe-Lintner case) proceed as follows. First of all, we will estimate ${ }^{\beta_{i}}$ for each asset $i=1, \ldots, N$ with $N$ fixed) from the linear regression, using the time series data and the average or excessive yield for each asset, as well as the error variation. Second, we estimate the regression of the second stage using the cross-section of the average yields and the error variation. If $\hat{X}$ is the matrix ${ }^{N x} 4$ which contains a column of them, a column $\hat{\beta}_{i}$, a column $\hat{\beta}_{i}^{2}$ and a column ${ }^{\hat{\sigma}_{s i}}$, then we come to the relationship:

$\widehat{\Gamma}=\left(\hat{\gamma}_{0}, \hat{\gamma}_{1}, \hat{\gamma}_{2}, \hat{\gamma}_{3}\right)^{T}=\left(\hat{X}^{T} W \widehat{X}\right)^{-1} \hat{X}^{T} W \bar{Z}=\frac{1}{T} \sum_{t=1}^{T} \widehat{\Gamma}_{t} ; \quad \widehat{\Gamma}_{t}=\left(\hat{X}^{T} W \hat{X}\right)^{-1} \hat{X} W Z_{t}$

least estimated OLS of $\Gamma_{t}=\left(\gamma_{0}, \gamma_{1}, \gamma_{2}, \gamma_{3}\right)^{T}$.

Third, we test the hypothesis that $\gamma_{j}=0, j=2,3$ using $t$ or Wald statistics such as (46) but with $\tilde{\Gamma}$ replaced by $\widehat{\Gamma}$. The main problem is that, in general, standard FM errors are incorrect because they ignore the errors in the variables or the regressor-generating problem, caused by the use of estimated quantities. $\hat{\beta}_{i}$ and $\hat{\sigma}_{i i}$. This leads to estimates $\Gamma$ are positive in small samples, but standard errors are inconsistent when applied to individual stocks.

The problem of the generated regressors was studied in Pagan (1984). Shanken (1992) proposes an analytical correction for these situations, showing that the asymptotic variation is correct in the case of high $T$ and small $N$. On the other hand, if the cross-section is composed of diversified portfolios, consisting of a large number of basic assets, it turns out that standard FM errors and Wald statistics may be correct, because in this case it is estimated that the items in the portfolio are accurately estimated, that is the measurement error $\hat{\beta}_{i}$ is small.

In fact, a key element of FM methodology is the use of a sophisticated portfolio group based on different samples to address the issue of measurement errors and other statistical problems. The complete FM algorithm is achieved through:

- Estimation of the market model to obtain the beta level of safety for $n$ stocks in period $A$ (portfolio formation period), approximately seven years. The securities are sorted into $\mathrm{N}$ test portfolios (twenty portfolios equally weighted based on the individually classified beta level) containing an approximately equal number of securities;

- Estimating the market model of the individual securities in period B (estimation period), approximately in the next five years, in order to obtain the level of beta security and standard deviations;

- Finally, the calculation based on regression and cross-section according to formula (38) using the portfolio returns from period $C$ (testing period), approximately the next four years, and the beta levels from the portfolio, etc., calculated as a corresponding linear combination. the individual beta levels in period $B$. The null hypothesis is tested using $t$ statistics or the Wald test, according to the relation (46).

The five-year estimation period is chosen taking into account some possible non-stationary beta and alpha levels. Portfolio beta is estimated from separate periods of five years as a result of the regression phenomenon. High beta estimates are associated with high positive measurement errors and vice versa, leading to an overestimation of the true beta level. Portfolio testing instead of individual stocks can alleviate the problem of empirical errors, because the estimation ones cancel each other out. Subsequent analyzes used double-sorted portfolios, including a secondary feature, such as market capitalization. Performing tests using data from period $\mathrm{C}$ aims to make the measurement error from the estimation of 
period $B$ independent of the terms of the error in the cross-sectional regression. Nine different periods (ie nine different periods $A$, nine different periods $B$ and nine different periods $C$ ) were considered. According to different data filters, there were between 435 and 845 securities used during each period.

Empirically, it has been found that there is a positive and linear relationship between beta risk yield and $R^{2}$ high, but $\gamma_{0}>0$ and $\gamma_{1}$ were significantly lower than the excessive market yield. Table 1 shows that, on average, over the period $\gamma_{i t}$ don't reject hypotheses. When $\hat{\sigma}_{s i}$ is contrary to $t$ statistics for $\gamma_{1}$ it becomes significant over the whole period, but the results on subperiods are not as good. The FM suggests that this is due to the substantial variability from month to month of the parameters. The FM also finds that this behavior over time $\widehat{\gamma}_{1 t}, \hat{\gamma}_{2 t}, \hat{\gamma}_{3 t}$ are in line with the market efficiency hypothesis, ie its autocorrelation

function $\hat{\gamma}_{i t}$ it is close to statistical zero, although this finding does not seem to be robust for the sample period or for the presence of explanatory variables. FM provides evidence that portfolio grouping effectively reduced the measurement error issue, as the average standard deviation of portfolio regression errors was on the order of one-third of the individual regression parameters of the stocks. It has moved on to multifactorial models following Fama's opinions.

\section{Conclusions}

Analyzing the aspects included in this article, the main aspects regarding the testing of the opaque version of the CAPM, some conclusions are drawn, namely. There is a cross equation constraint under the conditions of the null hypothesis and thus the model is restricted, it must be estimated under nonlinearity conditions. The unconstrained model is estimated under the variant that before using the total yields we must use the excess yields to eliminate the evolutionary peaks.

Another conclusion is that CAPM can provide good solutions, but sometimes weaker than others, because estimating maximum likelihood implies a normal multivariate yield.

If we use some statistics we come to the conclusion that robustness and normality must be interpreted in close correlation with the heterostedasticity of the time series. Also, in the analysis of the opaque version (black) we must take into account the cross-regression tests that were based on Fama and MacBeth 1973, which introduced the two-regression approach, which makes this research easy and then relatively easy to solve and implemented. Such an approach underscores the cross-cutting implications of CAPM, as Sharpe-Lintner has pointed out, specifying that the firm with excessive returns should be linearly linked to the firm with a slope equal to excessive average performance on the market. In the case of opaque, the average yield of the company should be linearly linked to another firm with the cross-sectional value and a slope equal to the excessive average yield on the market compared to the zero beta portfolio.

The data lead to the conclusion that for a correct analysis a test and the concept of the CAPM model must be performed before using it to ensure that the results are guaranteed with a high probability.

\section{References}

1. Ameur, H. B., Prigent, J. L. (2010). Behaviour towards Risk in Structured Portfolio Management. International Journal of Economics and Finance, 2 (5), 91-102

2. Ang, A., Hodrick, R., Xing, Y., Zhang, X. (2006). The cross section of volatility and expected returns. Journal of Finance, 61, 259-299

3. Anghel, M. G. (2013). Modele de gestiune şi analiză a portofoliilor, Editura Economică, Bucureşti

4. Anghelache, C., Anghel, M. G., lacob, Ș. V., Bîrsan, O. (2019). Analysis of the Effect of Acceleration in Dynamic Models. International Journal of Academic Research in Accounting, Finance and Management Sciences, 9 (4), 43-48

5. Anghelache, C., Anghel, M. G., Popovici, M. (2016a). Model privind managementul dinamic al portofoliului de acţiuni / Model regarding the dynamic management of shares portfolio. Romanian Statistical Review, Supplement, 7, 87-93

6. Anghelache, C. (2008). Tratat de statistică teoretică şi economică, Editura Economică, Bucureşti 
7. Anghelache, G. V., Anghel, M. G., Bodo, G. (2016b). Model of Static Portfolio Choices in an ArrowDebreu Economy. Romanian Statistical Review Supplement, 1/2016, 58-62

8. Campbell, J. V., Viceira, L. M. (2002). Strategic asset allocation: Portofolio choice for long-term investors. Clarendon lectures in economics, Oxford University Press

9. Chen, J., Ang, A. (2007). CAPM. Over the long-run: 1926-2001. Journal of Empirical Finance, 14, 1, $1-40$

10.Gollier, C. (2011). Portfolio Choices and Asset Prices: The Comparative Statics of Ambiguity Aversion. The Review of Economic Studies, 78 (4), 1329-1344

11.Grauer, R.R., Janmaat, J.A. (2009). On the power of cross-sectional and multivariate tests of the CAPM. Journal of Banking\&Finance, 33 (5), 775-787

12.lacob, S..V., Dumitru, D., Popovici, M. (2020). The main issues regarding the choice of portfolio and the testing of the model regarding the price of capital assets. Romanian Statistical Review Supplement, 2, $39-50$

13.Tetlock, P. C. (2011). All the News that's Fit to Reprint: Do Investors React to Stale Information?, Review of Financial Studies, 24, 1481-1512

14.Wachter, J. (2002). Portofolio and consumption decisions under mean-reverting returns: An exact solution for complete markets. Journal of Financial and Quantitative Analysis, 37 (1), 63-91

15.Wolff, E. (2013). The Asset Price Meltdown and the Wealth of the Middle Class, NBER Working Paper No. 18559

16. Wooldrige, J. (2006). Introductory econometrics. A modern approach - 2 edition, MIT Press

17.Zhou, G. (2010). How much stock return predictability can we expect from an asset pricing model?, Economics Letter, 108, 184-186. 